\title{
Validation of COI metabarcoding primers for terrestrial arthropods
}

\author{
Vasco Elbrecht ${ }^{\text {Corresp., } 1}$, Thomas WA Braukmann ${ }^{1}$, Natalia V Ivanova ${ }^{1,2}{ }^{2}$, Sean WJ Prosser ${ }^{1}$, Mehrdad Hajibabaei $^{1,2}$, \\ Michael Wright ${ }^{1,2}$, Evgeny V Zakharov ${ }^{1,2}$, Paul DN Hebert ${ }^{1,2}$, Dirk Steinke ${ }^{1,2}$ \\ ${ }^{1}$ Centre for Biodiversity Genomics, University of Guelph, Guelph, ON, Canada \\ 2 Department of Integrative Biology, University of Guelph, Guelph, ON, Canada \\ Corresponding Author: Vasco Elbrecht \\ Email address: luckylion07@googlemail.com
}

Metabarcoding can rapidly determine the species composition of bulk samples and thus aids biodiversity and ecosystem assessment. However, it is essential to use primer sets that minimize amplification bias among taxa to maximize species recovery. Despite this fact, the performance of primer sets employed for metabarcoding terrestrial arthropods has not been sufficiently evaluated. This study tests the performance of 36 primer sets on a mock community containing 374 species. Amplification success was assessed with gradient PCRs and the 21 most promising primer sets selected for metabarcoding. These 21 primer sets were also tested by metabarcoding a Malaise trap sample.

We identified eight primer sets, mainly those including inosine and/or high degeneracy, that recovered more than $95 \%$ of the species in the mock community. Results from the Malaise trap sample were congruent with the mock community, but primer sets generating short amplicons produced potential false positives. Taxon recovery from both mock community and Malaise trap sample metabarcoding were used to select four primer sets for additional evaluation at different annealing temperatures (40-60 $\left.\mathrm{C}^{\circ}\right)$ using the mock community. The effect of temperature varied by primer pair but overall it only had a minor effect on taxon recovery.

This study reveals the weak performance of some primer sets employed in past studies. It also demonstrates that certain primer sets can recover most taxa in a diverse species assemblage. Thus, based our experimental set up, there is no need to employ several primer sets targeting the same gene region. We identify several suitable primer sets for arthropod metabarcoding, and specifically recommend $B F 3+B R 2$, as it is not affected by primer slippage and provides maximal taxonomic resolution. The fwhF2 + fwhR2n primer set amplifies a shorter fragment and is therefore ideal when targeting degraded DNA (e.g. from gut contents). 
1 Title: Validation of COI metabarcoding primers for terrestrial arthropods

3 Running Title: COI Primers for arthropod metabarcoding

4 Word count:

5 Authors: Vasco Elbrecht ${ }^{1 *}$, Thomas WA Braukmann ${ }^{1}$, Natalia V Ivanova ${ }^{1,2}$, Sean WJ Prosser ${ }^{1}$, 6 Mehrdad Hajibabaei ${ }^{1,2}$, Michael Wright ${ }^{1,2}$, Evgeny V Zakharov ${ }^{1,2}$, Paul DN Hebert ${ }^{1,2}$, Dirk 7 Steinke $\mathrm{e}^{1,2}$

9 Affiliations:

10 1) Centre for Biodiversity Genomics, University of Guelph, 50 Stone Road East, Guelph, Ontario,

11 N1G 2W1, Canada

2) Department of Integrative Biology, University of Guelph, 50 Stone Road East, Guelph, Ontario, N1G 2W1, Canada

*Corresponding author: Vasco Elbrecht (elbrecht@uoguelph.ca), 


\section{Abstract}

Metabarcoding can rapidly determine the species composition of bulk samples and thus aids biodiversity and ecosystem assessment. However, it is essential to use primer sets that minimize amplification bias among taxa to maximize species recovery. Despite this fact, the performance of primer sets employed for metabarcoding terrestrial arthropods has not been sufficiently evaluated. This study tests the performance of 36 primer sets on a mock community containing 374 species. Amplification success was assessed with gradient PCRs and the 21 most promising primer sets selected for metabarcoding. These 21 primer sets were also tested by metabarcoding a Malaise trap sample.

We identified eight primer sets, mainly those including inosine and/or high degeneracy, that recovered more than $95 \%$ of the species in the mock community. Results from the Malaise trap sample were congruent with the mock community, but primer sets generating short amplicons produced potential false positives. Taxon recovery from both mock community and Malaise trap sample metabarcoding were used to select four primer sets for additional evaluation at different annealing temperatures $\left(40-60 \mathrm{C}^{\circ}\right)$ using the mock community. The effect of temperature varied by primer pair but overall it only had a minor effect on taxon recovery.

This study reveals the weak performance of some primer sets employed in past studies. It also demonstrates that certain primer sets can recover most taxa in a diverse species assemblage. Thus, based our experimental set up, there is no need to employ several primer sets targeting the same gene region. We identify several suitable primer sets for arthropod metabarcoding, and specifically recommend $\mathrm{BF} 3+\mathrm{BR} 2$, as it is not affected by primer slippage and provides maximal taxonomic resolution. The fwhF2 + fwhR2n primer set amplifies a shorter fragment and is therefore ideal when targeting degraded DNA (e.g. from gut contents). 


\section{Introduction}

45

Over the past decade, two methodological and technological advances have made it possible to address the urgent need for the capacity to undertake large-scale surveys of biodiversity (Vörösmarty et al. 2010; Dirzo et al. 2014; Steffen et al. 2015). First, the emergence of DNA barcoding which uses sequence variation in short, standardized gene regions (i.e. DNA barcodes) to discriminate species, has made it possible to quickly and reliably characterize species diversity (Hebert et al. 2003). Second, high-throughput sequencers (HTS) permit the inexpensive acquisition of millions of sequence records (Reuter et al. 2015). The coupling of HTS with DNA barcoding, commonly known as metabarcoding, allows for characterization of biodiversity at unprecedented scales (Creer et al. 2016) as shown by studies of terrestrial (Gibson et al. 2014; Beng et al. 2016), freshwater (Hajibabaei et al. 2011; Carew et al. 2013; Andújar et al. 2017), and marine (Leray \& Knowlton 2015) ecosystems.

Metabarcoding studies on bulk collections of animals usually target a subset of the $658 \mathrm{bp}$ cytochrome $c$ oxidase subunit I (COI) "Folmer" region (Folmer et al. 1994; Andújar et al. 2018). This gene region has gained broad adoption because of a rapidly expanding reference database (Ratnasingham \& Hebert 2007; Porter \& Hajibabaei 2018b) and its good taxonomic resolution (Meusnier et al. 2008). Ribosomal markers have been suggested as an alternative (Deagle et al. 2014; Marquina et al. 2018) because their slower rate of evolution results in more conserved motifs/regions aiding the design of universal primer sets. However, arthropod reference databases for ribosomal markers are very limited for most taxonomic groups (Clarke et al. 2014) and ribosomal primer sets often show no substantial improvement in taxon recovery over well- 
65 designed COI primer sets (Elbrecht et al. 2016; Clarke et al. 2017; Elbrecht \& Leese 2017;

66 Krehenwinkel et al. 2017).

An important consideration for metabarcoding studies is the primer combination used for amplification of the target fragment. It is critical that primer sets optimally match the template sequences of the target species. Mismatches between primer and template can skew read abundance and lead to a substantial bias in taxon detection (Piñol et al. 2014; Elbrecht \& Leese 2015). Failure to minimize amplification bias reduces the amount of taxa detected in a sample (Elbrecht \& Leese 2017). Furthermore, insufficient sequencing depth and/or low DNA concentration can introduce stochastic effects that additionally bias taxon recovery (Barnes \& Turner 2015; Leray \& Knowlton 2017).

The effectiveness of primer sets can be evaluated by in vitro tests with mock communities (Elbrecht \& Leese 2015; Brandon-Mong et al. 2015; Leray \& Knowlton 2017) or by in silico tests (Clarke et al. 2014; Elbrecht \& Leese 2016; Piñol et al. 2018; Bylemans et al. 2018b; Marquina et al. 2018). The failure to evaluate primers can seriously compromise data quality. For instance, a primer set (Zeale et al. 2011) often employed for analyzing gut contents of insect predators (see references in Jusino et al. 2018) lacks degeneracy, leading to poor taxon recovery (Brandon-Mong et al. 2015). The use of multiple primer sets or even mulinle marker genes was proposed to improve taxon recovery (Alberdi et al. 2017; Zhang et al. 2018). This approach may be optimal for samples of very phylogenetically divergent groups such as protists (Pawlowski et al. 2012) or marine benthic communities (Cowart et al. 2015; Wangensteen et al. 2018; Drummond 2018). However, given the increased cost and time associated with amplifying and sequencing additional markers (Bohmann et al. 2018; Zhang et al. 2018), the use of multiple primer sets is unnecessary for taxonomic groups with limited diversity. Additionally, relative read abundance comparisons 
88

between primer sets will become difficult, as each is subjected to different primer biases (Elbrecht \& Leese 2015). We hypothesize that in the case of terrestrial arthropods a single well-designed primer set can be sufficiently effective, and the use of multiple primer sets is not necessary.

This study compares the performance of commonly used and newly developed primer sets on the recovery of species in a bulk DNA extract from 374 insect species (Braukmann et al. 2019) and from a Malaise trap sample. Based on a hierarchical testing scheme (Figure 1) using gradient PCRs and assessing species recovery with metabarcoding, we selected four primer pairs whose metabarcoding performance was tested on a range of annealing temperatures.

\section{Material and Methods}

\section{Tested samples and experimental outline}

We used two samples to test a range of primer sets for metabarcoding: a mock community of 374 species (Braukmann et al. 2019) and a sample collected with a Malaise trap (Figure 1). The mock community is comprised of 374 species (Figure 1A), each specimen represented by an individual BIN (taxonomic breakdown shown in Figure S1A, (Ratnasingham \& Hebert 2013)). A detailed list of specimens and their Barcode of Life Datasystems process IDs (BOLD, Ratnasingham \& Hebert 2007) is given in Table S1. For most specimens, the full 658 bp barcode region was available through BOLD, but we completed reads for three taxa with shorter sequences by extracting haplotypes from our metabarcoding data using a denoising approach (Elbrecht et al. 2018b). The resulting reference library is available as a fasta file (See Scripts S1). To compare mock community results with a field sample, we collected insects with a Townes-style Malaise trap (Bugdorm, Taiwan) deployed in a grassland/forest area near Waterloo, Ontario, Canada $\left(43^{\circ} 29^{\prime} 30.8^{\prime \prime} \mathrm{N} 80^{\circ} 36^{\prime} 59.6^{\prime \prime} \mathrm{W}\right)$. We selected a single weekly sample (June 30 - July 7, 2018) and 
111 dried it for three days in a disposable grinding chamber. The sample was ground to fine powder

112 using an IKA Tube Mill control (IKA, Breisgau, Germany) at 25,000 rpm for $2 \times 3$ minutes. DNA

113 was extracted from $21 \mathrm{mg}$ of ground tissue using the DNeasy Blood \& Tissue kit (Qiagen, Venlo,

114 Netherlands).

115 The mock community DNA extract was used to test 36 primer pairs by comparing 116 amplification success across a range of annealing temperatures. Twenty-one primer pairs whose 117 amplicon concentrations plateaued in amplicon concentration at lower annealing temperatures 118 were selected for metabarcoding both the mock community and the Malaise trap sample. Four 119 representative primer sets showing high success in species recovery were selected to determine the 120 optimal annealing temperature for maximizing species recovery from bulk samples (see Figure 1).

\section{Gradient PCRs}

Thirty-six primer combinations commonly used for metabarcoding were selected for gradient PCR (Figure 2), to assess primer efficiency at different annealing temperatures. Some of these primers represent new combinations, as well as new variants of primers by shifting the primer binding site by $3 \mathrm{bp}$, by incorporating degeneracy, or by replacing inosine "I" with "N" and vice versa. PrimerMiner v0.18 was used to generate an alignment visualization (Elbrecht \& Leese 2016) using reference sequences for 31 arthropod orders downloaded and aligned as part of an earlier study (Vamos et al. 2017). The plot of the full alignment with binding sites for all primers used in this study is available in Figure S2.

Mock community gradient PCRs for 36 primer combinations were run on an Eppendorf 
134 molecular grade water (HyPure, GE, Utha, USA) for a total volume of $25 \mu \mathrm{L}$. One BF2 + BR2

135 primer (Elbrecht \& Leese 2017) positive control using the mock community DNA and one

136 negative control were included with each primer set.

137

138

139

140

141

142

143

144

145

146

147

148

149

150

151

152

153

154

155

156

The following thermocycling protocol was used: initial denaturation at $95^{\circ} \mathrm{C}$ for 5 min then 29 cycles of denaturation at $95^{\circ} \mathrm{C}$ for $30 \mathrm{~s}$ followed by a gradient of annealing temperatures from $44.5-64.5^{\circ} \mathrm{C}$ for $30 \mathrm{~s}$ with extension at $72^{\circ} \mathrm{C}$ for $50 \mathrm{~s}$, and a final extensions of $5 \mathrm{~min}$ at $72^{\circ} \mathrm{C}$. PCR success and fragment length were determined by visualizing amplicons on a 1\% agarose gel. Amplicon concentration was quantified without prior cleanup using a High Sensitivity dsDNA Kit on a Qubit fluorometer (Thermo Fisher Scientific, MA, USA).

3

\section{Primer selection for metabarcoding}

Based on the results of gradient PCR (Figure 1B), we selected 21 primer sets for metabarcoding that showed strong, consistent amplification and reached plateau in amplicon concentration at lower annealing temperatures (minimum of $0.6 \mathrm{x}$ amplification at $50.2{ }^{\circ} \mathrm{C}$, Figure S8). A few primer sets generated amplicons at $46^{\circ} \mathrm{C}$, but were excluded because they failed to reach an asymptote in concentration at lower annealing temperatures.

\section{Metabarcoding (mock community and malaise trap)}

21 primer sets for both the mock community and the Malaise trap sample were selected for DNA metabarcoding and Illumina MiSeq sequencing. We employed a fusion primer based twostep PCR protocol that amplifies target fragments in the first step and attaches in-line tags and Illumina TruSeq library sequence tails during the second PCR (Elbrecht \& Steinke 2018). To ensure sufficient sequence diversity for sequencing we used in-line tags of different length to shift 
157

158

159

160

161

amplicons against each other and sequenced half of the samples in reverse orientation by swapping the Illumina P5 and P7 sequencing adapters on the fusion primers (Elbrecht \& Leese 2015). The 7 bp tags with different insert lengths were randomly generated using R scripts (Elbrecht \& Steinke 2018), but were subsequently manually edited to maximize the Levenshtein distance between tags (Figure S3). Figure S4 shows the fusion primer sequences used for library preparation.

For the first PCR step, we used the same protocol as for the gradient PCR but used a fixed annealing temperature of $46^{\circ} \mathrm{C}$ and 24 cycles of amplification. One negative control containing the $\mathrm{BF} 2+\mathrm{BR} 2$ primer combination and one containing no primers were included in the PCR (see Table S2 for primer list).

For the second PCR step, $1 \mu \mathrm{L}$ of the PCR product generated by each primer set was used as template (with no quantification or reaction cleanup) under similar PCR conditions to the first PCR step except the extension time was increased to 2 minutes while the number of cycles was reduced to 14 .

PCR products were cleaned using SPRIselect (Beckman Coulter, CA, USA) with a sample to volume ratio of $0.76 \mathrm{x}$. DNA concentration was quantified using a Qubit fluorometer, High Sensitivity dsDNA Kit (Thermo Fisher Scientific, MA, USA). Subsequently, individual libraries were equimolar pooled following adjustment for amplicon length (Table S1).

The mock community library was sequenced on an Illumina MiSeq with 300 bp paired end sequencing (v3 chemistry) with a 5\% PhiX spike in. Amplicons for the Malaise sample were generated with half the DNA amount $(6.25 \mathrm{ng})$ to reduce the chance of PCR inhibition and 29 cycles for the first PCR step. Individual libraries were pooled equimolar, but we factored in the preference for shorter reads by Illumina sequencing using the mock community sequencing results 
179 (Figure S9, Table S1). The Malaise sample was also sequenced on an Illumina MiSeq with 300 bp 180 paired end sequencing (v3 chemistry) with a 5\% PhiX spike in.

For both sequencing runs, PCR negative controls where omitted, as additional fusion primers would have been needed for sample tagging. For the same reason, we also decided against PCR replicates, given that DNA metabarcoding results are usually highly reproducible (Elbrecht et al. 2017; Braukmann et al. 2019) and potential tag switching could be easily identified based on the different primer sets used (i.e. amplicon length).

\section{Bioinformatic processing}

Quality control of raw sequence data was done with FastQC v0.11.7 and multiQC v1.4 (Ewels et al. 2016). Sequence data was first demultiplexed and processed with the R wrapper script JAMP v0.68 (https://github.com/VascoElbrecht/JAMP). Reads were paired-end merged using Usearch v11.0.667 (Edgar 2010), allowing for 99 mismatches or 75\% similarity between overlapping regions to maximize the amount of merged reads. Primer sequences were subsequently trimmed using cutadapt v1.18 with default settings (Martin 2011). Reads deviating by more than $10 \mathrm{bp}$ from the expected amplicon length were discarded. Usearch (Edgar \& Flyvbjerg 2015) was used to remove reads with an expected error probability of 1 or higher, and to dereplicate and map reads against the 374 reference sequences of the mock community (usearch_global with minimum 97\% identity). Resulting tables were automatically summarized into a hit table of all samples using the function map2ref implemented in JAMP. The hit table was subsampled using a custom R script (Scripts S1) to determine the number of taxa detected at different sequencing depths. Figure 3 provides an overview of the results of the processing steps and all scripts are available in Scripts S1. 
Data for the Malaise sample was processed using the same pipeline but mapped against a

reference database consisting of public sequence records for arthropods found in Ontario (downloaded from BOLD December 2018). Gaps and terminal Ns were removed from all sequences. Sequences outside the length range of 648-668 bp were discarded (Scripts S1). Reads were mapped against this reference database using map2ref, but singletons in each sample were discarded and mapping required a $99 \%$ match and maxaccepts $=0$, maxrejects $=0$, to reduce the number of false positives. Reads matching to the same Barcode Index Number (BIN, Ratnasignham \& Hebert 2013) were collapsed and reads that matched reference sequences that lacked a BIN assignment were merged based on taxonomy, and combined into 11 MOTUs.

\section{Gradient metabarcoding}

Out of the 21 metabarcoded primer sets, we selected four representative primer sets of different amplicon length that recovered over 95\% the mock community (ArF5 + Fol-degen-rev, $\mathrm{BF} 3+\mathrm{BR} 2$, mlCOIintF + Fol-degen-rev and fwhF2 + fwhR2n, Figure 1D) to evaluate the impact of nine annealing temperatures $\left(40.0,41.6,43.7,46.0,48.5,50.8,53.0,54.7\right.$ and $\left.56.0{ }^{\circ} \mathrm{C}\right)$ on taxon recovery. Temperatures below $46^{\circ} \mathrm{C}$ were specifically chosen to explore the impact of non-specific amplification. Other than running the first and second PCR step as gradient PCRs, all laboratory conditions and bioinformatic steps were identical to the prior mock community metabarcoding run. For tagging samples in the second PCR step, additional fusion primers were developed (Fig S5) and checked for sufficient Levenshtein distance (Fig S6, (Elbrecht \& Steinke 2018)). Individual samples were equimolar pooled, and the library sequenced using an Illumina MiSeq with 300 bp paired end sequencing (v3 chemistry) and a 5\% PhiX spike in. Bioinformatic analysis was identical to the previous mock community MiSeq run at $46^{\circ} \mathrm{C}$ annealing temperature. 
226 Statistical analysis

For statistical analysis $\mathrm{R}$ v3.5.0 was used and all scripts used to generate figures are available in Scripts S1. To simulate the effect? of equal sequencing depth at 10,000 and 100,000 reads with each primer set, hit tables were subsampled 1,000 times and number of BINs or taxa recovered was averaged. The relative abundance of reads per taxon (above $0.001 \%$ ) for each of 231 the 21 primer sets (Table S1) tested with the mock community was analysed using a Principal 232 Component Analysis implemented in the R package FactoMineR v1.34. The same data was used 233 to visualize the similarity between communities recovered with each primer set, using the $\mathrm{R}$ 234 package vegan v2.5-2. A dendrogram was generated using both Jaccard similarity and Bray-Curtis 235 dissimilarity. 
236

237

\section{Results}

\section{Gradient PCR results and primer set selection}

All primer sets generated amplicons with the expected length (Fig. S7) although a few amplicons showed faint secondary bands following the gradient PCR. None of the negative controls showed visible bands. Amplicon concentrations $(\mathrm{ng} / \mu \mathrm{l})$ reached an asymptote for 21 of the 36 primer sets $(58 \%)$ at $<50^{\circ} \mathrm{C}$ and they were selected for sequencing (Fig. S8). While some other primers showed clear bands in the agarose gel (Fig. S7), they were excluded from sequencing because of their limited annealing temperature range.

Amplification success for newly designed primers showed mixed results (Fig. S7 \& Fig. S8). A more degenerate version of ZBJ-ArtF1c $+\mathrm{ZBJ}-\mathrm{ArtR} 2 \mathrm{c}$ decreased amplification efficiency. Substituting N for inosine led to increased amplification efficiency for BF1i+BR1i, while replacing inosine with $\mathrm{N}$ reduced amplification efficiency for $\mathrm{Bn}+\mathrm{En}$. The binding site of the BF2 primer was shifted 3 bp forward (BF3) to reduce slippage effects (Elbrecht et al. 2018a). In combination with the BR2 primer set, both versions showed similar amplification efficiency.

\section{Metabarcoding and bioinformatic processing}

Sequencing of the mock community tested with 21 primer sets on MiSeq (300 bp PE) produced $24,348,000$ reads of good quality $(\mathrm{Q} 30=<85.8 \%$ of reads). The PCR negative control (not included in sequencing) showed no visible band on a agarose gel. Raw sequence data is available on NCBI SRA via accession number SRX4908948. Sequencing depth was negatively correlated with amplicon length (Fig. S9, linear regression, $\mathrm{p}<0.0001$ ), with at least $280 \mathrm{~K}$ sequences per sample. The number of discarded sequences after data processing varied among primer sets (Fig. 3A); on average $80.61 \%(\mathrm{SD}=9.84 \%)$ of the reads were mapped to the 374 
259 reference sequences. For the primer sets MZplankF2 + C_LepFolR, BF3 + BR2, BF2 + BR2 and 260 AncientLepF3 + C_LepFolR more than 3\% of the amplicons deviated by more than $10 \mathrm{bp}$ from 261 the expected amplicon length (Fig. 3, Fig. S10). Primer combinations involving mlCOIintF, BF1, 262 S1.

The Malaise sample yielded $16,629,020$ reads of good quality (Q30=<92.59\% of reads). 
282 lacked degeneracy often had low amplification success and detected fewer species than primer sets 283 with degeneracy (Fig. S12). The primer combinations fwhF2 + fwhR2n, BF1/BF2/BF3 + BR2,

284 285 286 287 288 289

ArF5 + Fol-degen-rev and those based on mlCOIintF and its derivatives showed the best performance with similar recovery rates (recovery $\geq 95 \%$ of the community, Fig.4, Fig. S12). Taxa recovery was consistent across orders, except for Hymenoptera which were often recovered with lower read counts (Fig. S1A). A Principal Component Analysis (PCA) of relative taxon recovery shows that primer combinations with similar taxon recovery tended to cluster together (Fig. S13), although only $29.36 \%$ of variability can be explained by both components. Jaccard similarity and Bray-Curtis based dendograms (Fig. S14) illustrate that recovery is generally similar among primers, but that combinations with poor species recovery tend to cluster together.

Sequencing of the Malaise sample confirmed the strong performance of some primer sets, but others showed lower species recovery (Figure 4B). As the species composition of the Malaise sample was unknown, BIN counts at different sequencing depths were used to estimate taxon recovery for all sets of primers. Heat maps for both the Malaise sample (Fig. S18) and the mock community (Fig. S12) were generally congruent but short amplicons from the Malaise sample detected more taxa present in very low abundance. This trend was also reflected in the number of taxa detected with each primer set (Figure 4B) because longer amplicons such as Ill_B_F + HCO2198, AcientLepF3 + C_LepFolR or LCO1490 + HCO2198 exhibited lower taxon recovery than shorter fragments. Most primers that performed well for the mock community also did so for the Malaise sample (highlighted in green in Figure 4B), except the ArF5 + Fol-degen-rev primer set. These patterns were consistent with varying sequencing depths with no asymptote reached in the rarefaction analysis (Fig. S19). Additionally, the rarefaction analysis shows a greater range in the number of taxa detected with different primer sets than for the mock community (Fig S11). 
Detection across orders was very consistent for primer sets that show good taxa recovery, while especially Hymenoptera and Hemiptera were underrepresented with primer sets recovering fewer $\operatorname{taxa}($ Fig S1B).

\section{Gradient PCR metabarcoding}

When the performance of the four good performing primer pairs was analyzed at nine annealing temperatures, 23,770,810 sequences (NCBI SRA; ID: SRX4908947) were obtained with good read quality $(\mathrm{Q} 30=<82.9 \%$ of reads). Sequence coverage averaged 0.58 million $(\mathrm{SD}=0.1$ million) per sample with a lowest value of 0.38 million reads. The PCR negative control was not included in sequencing but it showed no visible band on the agarose gel. Results at $46^{\circ} \mathrm{C}$ were very similar to the prior metabarcoding run with abundance differences mostly affecting low abundant OTUs (Figure S15, linear regression adj. $\mathrm{R}^{2}>0.97$ ). Changes in annealing temperature from 40 $56^{\circ} \mathrm{C}$ only had minor effects on species recovery (Figure 5). In particular, two primer sets $(\mathrm{mlCOIintF}+$ Fol-degen-rev; fwhF2 + fwhR2n) showed little variation in species recovery across the range of annealing temperatures. By comparison, recovery rates decreased at temperatures above $\sim 53^{\circ} \mathrm{C}$ for both BF3 $+\mathrm{BR} 2$ and ArF5 + Fol-degen-rev (Figure 5, Figure S16). Length variation in amplicons as a result of primer slippage was not temperature dependent, but the $\mathrm{BF} 3+\mathrm{BR} 2$ primer set generated more short non-target amplicons at lower temperatures (over 1/4 of sequences, Figure S17).

\section{Discussion}

Using a mock community, we tested a total of 36 different primer combinations, 21 of which were selected for a more detailed metabarcoding analysis. While we did not run replicates for most 
328 primer sets, results at $46^{\circ} \mathrm{C}$ for gradient metabarcoding and the mock community run were similar.

329 This result is consistent with previous studies on bulk samples which indicated that replicates 330 typically produce similar results (Elbrecht et al. 2017; Braukmann et al. 2019), particularly when 331 the variation of low abundant OTUs (i.e. $<0.001 \%)$ introduced by stochastic effects is ignored 332 (Leray \& Knowlton 2017). Consequently, for metabarcoding of bulk samples, replication should 333 be done at the sampling level (Hurlbert 1984) rather than using DNA extracts or replicate PCRs. 334 While technical replicates do increase confidence in experimental outcomes and make it easier to 335 336 detect cross-contamination (Zepeda-Mendoza et al. 2016; Elbrecht \& Steinke 2018; Macher \& Weigand 2018), they deliver limited information given the substantial increase in cost and laboratory workload. If the detection of rare taxa is important for a project, an increase in sequencing depth (Smith \& Peay 2014; Braukmann et al. 2019) and use of a tagging system resistant to tag switching (e.g. fusion primers, Elbrecht et al. 2017) is a good alternative to replication. Even with the shallow sequencing depth (100,000 reads) used in this study, most primer sets recovered a majority of the taxa in the mock community. This was not necessarily the case for the Malaise trap sample (Figure S19) which is more diverse than the mock community tested. However, the comparison of taxon recovery at different sequencing depths by the tested primer sets allowed for good benchmarking, without capturing the full community. We were also able to characterize the positive bias of the Illumina MiSeq towards shorter fragments (Figure S which can be offset by adjusted amplicon concentrations when running fragments of different length in the same run (Fig. S9). 
350 As several primer sets recovered most of the taxa in the mock community in similar proportions

351 (Figure 4A), our study has identified several suitable primer sets for metabarcoding terrestrial 352 arthropods communities. The exact choice of primer set will depend on the context of a study, 353 required amplicon length and desired taxonomic resolution (Meusnier et al. 2008; Porter \&

354 355 356 Hajibabaei 2018a). For instance, the fwhF2 + fwhR2n primer set produces a 205 bp amplicon that is ideal when targeting degraded DNA in eDNA or gut contents (Bylemans et al. 2018a). The BF1 + BR2 and all three mlCOIintF-based primer sets generate slightly longer fragments (316/313 bp), but they are prone to slippage (Elbrecht et al. 2018a) which can cause problems with sequence denoising (Callahan et al. 2017) during data analysis. We overcame this problem for the longer $\mathrm{BF} 2+\mathrm{BR} 2$ fragment (421 bp) by moving the BF2 primer 3 bp forward (BF3). The BF3 + BR2 combination as well as the ArF5 + Fol-degen-rev primer set represent good choices for long (>400 bp) COI fragment amplification. The ArF5 + Fol-degen-rev primer set appears to be less affected by non-specific amplification at lower annealing temperatures than the BF3 + BR2 primer pair. Although these longer fragments can improve taxonomic resolution, they show less overlap in Illumina paired end sequencing leading to more reads being excluded during quality filtering (Figure 3).

We observed an increase of rare taxa detected with short amplicons in the Malaise sample, but these are likely false positives due to the decreased taxonomic resolution of shorter amplicons (Meusnier et al. 2008; Porter \& Hajibabaei 2018a). Even though the ZBJ-ArtF1c + ZBJ-ArtR2c primer set detected over 700 taxa in the Malaise sample, a value comparable to other well performing primer pairs, it failed to detect abundant BINs that most of the other primer pairs recovered (Fig. S18). Well performing primers showed no bias against specific orders, while less suitable primers did struggle with detection of Hymenoptera and Hemiptera. The decreased 
373 detection of Hymenoptera in the mock community can likely be attributed to the lysis protocol

374 used for DNA extraction from the insect abdomens (Braukmann et al. 2019). This was not the case

375 for the malaise sample, where the bulk sample was ground to a fine powder, making the tissue

376 more accessible to the lysis buffer.

377

378

379

380

381

382

383

384

385

386

387

388

389

390

391

392

393

394

395

Based on the results of this study we recommend the BF3+BR2 primer set for metabarcoding of malaise trap samples. The 458 bp long amplicon offers excellent taxonomic resolution and, unlike many of the other tested primer sets, it is not being affected by primer slippage, making it ideal for denoising approaches. Longer amplicons also contain more information about genetic variability and haplotypes when using Exact Sequence Variants (ESVs). While about $20 \%$ of the reads generated with this primer set are lost during quality filtering, this can be easily overcome by using more recent Illumina platforms (e.g. NovaSeq) as they provide much higher throughput (Singer et al. 2019). However, primer selection might vary with study goal, targeted taxonomic groups, desired taxonomic resolution, DNA quality, and sequencing platform used.

\section{Primer design}

Primer sets with differing degeneracy, inosine inclusion, and differences in the primer binding region showed variable taxonomic recovery making it difficult to establish clear predictors for primer performance. While degeneracy generally improves the universality of a primer (Krehenwinkel et al. 2017), some highly degenerate primers performed poorly in our tests (Figure 4). Additionally, even if a primer set shows good taxon recovery, it can still be susceptible to dimerization, to non-specific amplification, or to primer slippage (Elbrecht et al. 2018a) (Figure S10). These complexities are difficult to predict in silico and it is important to validate 
metabarcoding primer sets in vitro using taxa and samples from the targeted ecosystems. For example, the BF2+BR2 primer set generated non-specific amplicons (often bacterial), which can become a serious complication for eDNA studies where target DNA is scarce (Macher et al. 2018; Hajibabaei et al. 2019b, Collins et al. 2019). High primer degeneracy will likely increase primer universality but decrease specificity. This is less problematic when metabarcoding DNA extracts from bulk specimen samples where target DNA predominates but can be different for environmental DNA samples.

The present study did not reveal if the use of inosine can reduce problems created by high primer degeneracy. Some primers modified with inosine performed well, but others did not. The same was true for highly degenerate primers. However, we did show that for the fusion primer system (Elbrecht \& Steinke 2018), primers employed in the second PCR step can be designed with "N" instead of inosine (Figure S5). This substantially reduces costs when large fusion primer quantities are needed for reliably tagging and sample multiplexing. Primer performance could be further improved by adding degeneracy and / or using inosine, but performance will suffer if too much degeneracy is added. Despite careful primer design following best practices (Abd-Elsalam 2003), primer performance can still vary in its suitability for the primer binding site. A primer that works well on paper, might still not work in vitro and we strongly recommend testing primers with a mock community or field sample.

\section{Annealing temperature}

While primer choice is critical for metabarcoding projects, PCR can also be biased by the polymerase used (Nichols et al. 2018), cycle number (Vierna et al. 2017; Krehenwinkel et al. 2017), GC content (Braukmann et al. 2019), inhibitors (Demeke \& Jenkins 2009; Sellers et al. 
419 2018), and annealing temperature (Aylagas et al. 2016; Clarke et al. 2017; Krehenwinkel et al.

420 2018). It is generally assumed that primers bind better at lower annealing temperatures as potential

421 mismatches between template and primer have less influence. While touchdown PCR does not

422 improve species recovery (Clarke et al. 2017), lower annealing temperatures slightly increase it

423 (Aylagas et al. 2016). Although it seems intuitive that lower annealing temperatures lead to better

424 taxonomic recovery, previous studies explored only a limited temperature range never going below

$42546^{\circ} \mathrm{C}$, likely due to the increased risk of non-specific amplification. We studied four representative

426 primer pairs at 9 different annealing temperatures across a wider range (gradient PCR from 40 -

$42756^{\circ} \mathrm{C}$ ) and were unable to find a universal effect of annealing temperature. BF3 + BR2, mlCOIintF

$428+$ Fol-degen-rev and fwhF2 + fwhR2n primer sets are largely unaffected by changes in annealing

429 temperature. On the other hand, recovery peaks at $48.5^{\circ} \mathrm{C}$ for the $\mathrm{ArF} 5+\mathrm{Fol}-\mathrm{degen}-\mathrm{rev}$ primer

430 set. For all four primer pairs, annealing temperatures between $46-50{ }^{\circ} \mathrm{C}$ are probably good choices

431 for metabarcoding. However, this highly depends on melting temperature $\left(\mathrm{T}_{\mathrm{m}}\right)$. It is advisable to

432 test newly designed metabarcoding primers across an annealing temperature gradient. However,

433 given that most tested primers did perform similarly well at temperatures usually used for 434 metabarcoding, sequencing gradient PCRs might not always be necessary. Running the four primer 435 pairs at temperatures below $46^{\circ} \mathrm{C}$ did not substantially increase taxa recovery, while for some 436 primers it also increased the risk of dimer amplification and occurrence of non-target DNA.

No need for multiple primer sets

Eight primer combinations (Figure 4, highlighted in green) each detected $95 \%$ or more of

the taxa present in the mock community, and most of them could therefore be suitable choices for metabarcoding studies targeting terrestrial arthropods. Of these, seven showed very good 
442 performance with the malaise trap sample. This is in stark contrast to earlier studies (Alberdi et al.

443 2017; Zhang et al. 2018) recommending the use of multiple primer sets to increase coverage. This

444 discrepancy can be explained by primer choice, because (Zhang et al. 2018) used LCO1490 and

445 HCO2198 primers which lack degeneracy, and (Alberdi et al. 2017) worked with gut content

446 samples; thus replicates might be substantially affected by stochastic effects resulting from low

447 DNA yield. Additionally, the primers used (ZBJ-ArtF1c + ZBJ-ArtR2c) by Alberdi et al. (2017)

448 performed poorly in our study. This particular primer combination (Zeale et al. 2011) is widely

449

450

451

452

453

454

455

456

457

458

459

460

461 462

463

used for metabarcoding studies (Jusino et al. 2018) but our results show substantial amplification bias, confirming the low taxon recovery observed before for this primer pair (Brandon-Mong et al. 2015). An alternative primer pair to analyze gut content from predators consuming insects could be the pair fwhF $2+$ fwhR2n because it shows better taxonomic recovery.

The use of COI primer sets with limited or no degeneracy such as in (Zhang et al. 2018;

Jusino et al. 2018) is not recommended. In general, careful primer design and validation (ideally using mock communities) cannot be replaced by the use of multiple COI primer sets (Alberdi et al. 2017; Zhang et al. 2018) or ribosomal markers (Deagle et al. 2014), given the increased workload of a multi marker/primer approach and the limited taxonomic resolution of ribosomal markers (Clarke et al. 2017; Marquina et al. 2018). These results were also recently confirmed by (Hajibabaei et al. 2019a), which showed that the use of multiple primer sets did not substantially improve taxa detection.

\section{Conclusions}


Our study demonstrates that the fwhF2 + fwhR2n, BF1, BF2, BF3 + BR2 and mlCOIintF based primer sets all perform well when metabarcoding terrestrial arthropod samples. We recommend fwhF2 + fwhR2n for amplification of degraded DNA samples such as gut contents, and $\mathrm{BF} 3+\mathrm{BR} 2$ when high taxonomic resolution is required. The BF3+BR2 primer set is also ideal for working with Exact Sequence Variants (ESVs), as it is not affected by primer slippage. For most of these primer sets, annealing temperatures of $46-50^{\circ} \mathrm{C}$ are ideal. The present study also reinforces the importance of careful primer validation using mock and field samples, especially when primer performance has not yet been evaluated for the taxonomic group under study. Based on our results, the use of multiple primer sets seems rarely justified as it increases laboratory effort without substantially improving taxon recovery. Our study sets the stage for standardized approaches for large-scale biodiversity analysis. However, given the vast diversity of arthropods in different sampling approaches and geographical regions, primer tests on additional samples are required to fully confirm our findings for large-scale surveys of arthropod diversity.

\section{Data availability}

Raw sequence data is available on the NCBI SRA archive; accession SRX4908948 provides data for the mock community, accession SRX5175597 for the Malaise sample, and accession SRX4908947 for the gradient PCR experiment. Demultiplexed read 1 and read 2 files are available for all sequencing runs under the accessions listed in Table S3. The JAMP bioinformatics pipeline is available on GitHub https://github.com/VascoElbrecht/JAMP with the used settings detailed in Scripts S1. Sequence alignments generated with PrimerMiner are available at Dryad DOI. 
Figure 1: Overview of the experimental design. The performance of 36 primer pairs was tested via gradient PCRs with a mock community of insects (A). The 21 pairs that showed best amplification results (B) were selected for further DNA metabarcoding runs utilizing both the mock community and a Malaise trap sample (C). Based on the metabarcoding results, four primer sets showing good performance were selected for a third test that examined the effects of varying annealing temperatures on taxon recovery and non-specific amplification (D). Based on all results, the optimal primer sets were designated $(\mathrm{E})$. 
community. The number for each primer pair on the x-axis corresponds with that in Figure 2. B: Proportion of sequences discarded by max expected errors $=1$ filtering using Usearch, plotted against the length of the target region (in bp). Red line indicates linear regression.

Figure 4: Bar plot showing the number of BINs recovered using metabarcoding with 21 primer pairs. The dark grey bar indicates subsampling at 10,000 reads while the light grey bar indicates subsampling at 100,000 reads per sample, each run with 1,000 replicates. Error bars show the standard deviation. Primer combinations affected by primer slippage (Elbrecht \& Steinke 2018) are marked with "PS". A: Mock sample data, with primer combinations highlighted in green that detected more than 350 of the 374 BINs, while those that recovered fewer than 310 BINS are highlighted in red. B: Malaise trap data - primer combinations highlighted in green detected more than 750 BINs while those highlighted in red detected less than 600 BINs.

Figure 5: Bar plot showing the number of BINs recovered from the mock community at different annealing temperatures. The dark grey bar indicates subsampling at 10,000 reads, while the light grey bar depicts subsampling at 100,000 reads per samples; both were run with 1,000 replicates. Error bars show the standard deviation. 


\section{Acknowledgements}

We thank all staff at the CBG who collected the samples employed to assemble the mock community, and Al Woodhouse from the Waterloo District School Board for his aid with Malaise trap sampling. We also thank the Optimist Club of Kitchener-Waterloo for access to their site at Camp Heidelberg. We are grateful to the three reviewers and editor who provided comments that improved our manuscript. This study was supported by funding through the Canada First Research Excellence Fund. It represents a contribution to the University of Guelph Food From Thought research program.

\section{Abd-Elsalam, K.A. (2003). Bioinformatic tools and guideline for PCR primer design. african} Journal of biotechnology, 2, 91-95-95.

Alberdi, A., Aizpurua, O., Gilbert, M.T.P. \& Bohmann, K. (2017). Scrutinizing key steps for reliable metabarcoding of environmental samples (A. Mahon, Ed.). Methods in Ecology and Evolution, 17, 730-14.

Andújar, C., Arribas, P., Gray, C., Bruce, K., Woodward, G., Yu, D.W. \& Vogler, A.P. (2017). Metabarcoding of freshwater invertebrates to detect the effects of a pesticide spill. Molecular Ecology.

Andújar, C., Arribas, P., Yu, D.W., Vogler, A.P. \& Emerson, B.C. (2018). Why the COI barcode should be the community DNA metabarcode for the metazoa. Molecular Ecology, 27, 39683975 .

Aylagas, E., Borja, A., Irigoien, X. \& Rodríguez-Ezpeleta, N. (2016). Benchmarking DNA Metabarcoding for Biodiversity-Based Monitoring and Assessment. Frontiers in Environmental Science, 3, 1809-12.

Barnes, M.A. \& Turner, C.R. (2015). The ecology of environmental DNA and implications for conservation genetics. Conservation Genetics, 1-17.

Beng, K.C., Tomlinson, K.W., Shen, X.H., Surget-Groba, Y., Hughes, A.C., Corlett, R.T. \& Slik, J.W.F. (2016). The utility of DNA metabarcoding for studying the response of arthropod diversity and composition to land-use change in the tropics. Scientific Reports, 1-13. 
Bohmann, K., Gopalakrishnan, S., Nielsen, M., Nielsen, L.D.S.B., Jones, G., Streicker, D.G. \& Gilbert, M.T.P. (2018). Using DNA metabarcoding for simultaneous inference of common vampire bat diet and population structure. Molecular ecology resources, 18, 1050-1063.

Brandon-Mong, G.J., Gan, H.M., Sing, K.W., Lee, P.S., Lim, P.E. \& Wilson, J.J. (2015). DNA metabarcoding of insects and allies: an evaluation of primers and pipelines. Bulletin of Entomological Research, 105, 717-727.

Braukmann, T.W.A., Ivanova, N.V., Prosser, S.W.J., Elbrecht, V., Steinke, D., Ratnasingham, S., deWaard, J.R., Sones, J.E., Zakharov, E.V. \& Hebert, P.D.N. (2019). Metabarcoding a Diverse Arthropod Mock Community. Molecular ecology resources, 1-41.

Bylemans, J., Furlan, E.M., Gleeson, D.M., Hardy, C.M. \& Duncan, R.P. (2018a). Does Size Matter? An Experimental Evaluation of the Relative Abundance and Decay Rates of Aquatic Environmental DNA. Environmental science \& technology, 52, 6408-6416.

Bylemans, J., Gleeson, D.M., Hardy, C.M. \& Furlan, E. (2018b). Toward an ecoregion scale evaluation of eDNA metabarcoding primers: A case study for the freshwater fish biodiversity of the Murray-Darling Basin (Australia). Ecology and Evolution, 58, 403-16.

Callahan, B.J., McMurdie, P.J. \& Holmes, S.P. (2017). Exact sequence variants should replace operational taxonomic units in marker-gene data analysis. 1-5.

Carew, M.E., Pettigrove, V.J., Metzeling, L. \& Hoffmann, A.A. (2013). Environmental monitoring using next generation sequencing: rapid identification of macroinvertebrate bioindicator species. Frontiers in zoology, 10, 1-1.

Collins, R.A., Bakker, J., Wangensteen, O.S., Soto, A.Z., Corrigan, L., Sims, D.W., Genner, M.J. and Mariani, S., 2019. Non-specific amplification compromises environmental DNA metabarcoding with COI. Methods in Ecology and Evolution, in press.

Clarke, L.J., Beard, J.M., Swadling, K.M. \& Deagle, B.E. (2017). Effect of marker choice and thermal cycling protocol on zooplankton DNA metabarcoding studies. Ecology and Evolution, 7, 873-883.

Clarke, L.J., Soubrier, J., Weyrich, L.S. \& Cooper, A. (2014). Environmental metabarcodes for insects: in silicoPCR reveals potential for taxonomic bias. Molecular ecology resources, 14, $1160-1170$.

Cowart, D.A., Pinheiro, M., Mouchel, O., Maguer, M., Grall, J., Miné, J. \& Arnaud-Haond, S. (2015). Metabarcoding Is Powerful yet Still Blind: A Comparative Analysis of Morphological and Molecular Surveys of Seagrass Communities (S. Mazzuca, Ed.). PloS one, 10, e011756226.

Creer, S., Deiner, K., Frey, S., Porazinska, D., Taberlet, P., Thomas, W.K., Potter, C. \& Bik, H.M. (2016). The ecologist's field guide to sequence-based identification of biodiversity (R. Freckleton, Ed.). Methods in Ecology and Evolution, 7, 1008-1018. 
Deagle, B.E., Jarman, S.N., Coissac, E., Pompanon, F. \& Taberlet, P. (2014). DNA metabarcoding and the cytochrome c oxidase subunit I marker: not a perfect match. Biology Letters, 10, 1-4.

Demeke, T. \& Jenkins, G.R. (2009). Influence of DNA extraction methods, PCR inhibitors and quantification methods on real-time PCR assay of biotechnology-derived traits. Analytical and Bioanalytical Chemistry, 396, 1977-1990.

Dirzo, R., Young, H.S., Galetti, M., Ceballos, G., Isaac, N.J.B. \& Collen, B. (2014). Defaunation in the Anthropocene. Science (New York, NY), 345, 401-406.

Drummond, A.J. (2018). Evaluating a multigene environmental DNA approach for biodiversity assessment. 1-19.

Edgar, R.C. (2010). Search and clustering orders of magnitude faster than BLAST. Bioinformatics, 26, 2460-2461.

Edgar, R.C. \& Flyvbjerg, H. (2015). Error filtering, pair assembly and error correction for nextgeneration sequencing reads. Bioinformatics, 31, 3476-3482.

Elbrecht, V. \& Leese, F. (2015). Can DNA-Based Ecosystem Assessments Quantify Species Abundance? Testing Primer Bias and Biomass-Sequence Relationships with an Innovative Metabarcoding Protocol (M. Hajibabaei, Ed.). PloS one, 10, e0130324-16.

Elbrecht, V. \& Leese, F. (2016). PrimerMiner: an R package for development and in silico validation of DNA metabarcoding primers. Methods in Ecology and Evolution, 8, 622-626.

Elbrecht, V. \& Leese, F. (2017). Validation and development of freshwater invertebrate metabarcoding COI primers for Environmental Impact Assessment. Frontiers in Environmental Science, 5, 1-11.

Elbrecht, V. \& Steinke, D. (2018). Scaling up DNA metabarcoding for freshwater macrozoobenthos monitoring. Freshwater biology.

Elbrecht, V., Hebert, P.D.N. \& Steinke, D. (2018a). Slippage of degenerate primers can cause variation in amplicon length. Scientific Reports, 1-5.

Elbrecht, V., Taberlet, P., Dejean, T., Valentini, A., Usseglio-Polatera, P., Beisel, J.-N., Coissac, E., Boyer, F. \& Leese, F. (2016). Testing the potential of a ribosomal 16S marker for DNA metabarcoding of insects. PeerJ, 4, e1966-12.

Elbrecht, V., Vamos, E.E., Steinke, D. \& Leese, F. (2018b). Estimating intraspecific genetic diversity from community DNA metabarcoding data. PeerJ, 6, e4644-13.

Elbrecht, V., Vamos, E., Meissner, K., Aroviita, J. \& Leese, F. (2017). Assessing strengths and weaknesses of DNA metabarcoding based macroinvertebrate identification for routine stream monitoring. Methods in Ecology and Evolution, 1-21.

Ewels, P., Magnusson, M., Lundin, S. \& Käller, M. (2016). MultiQC: summarize analysis results 
for multiple tools and samples in a single report. Bioinformatics, 32, 3047-3048.

Folmer, O., Black, M., Hoeh, W., Lutz, R. \& Vrijenhoek, R. (1994). DNA primers for amplification of mitochondrial cytochrome $\mathrm{c}$ oxidase subunit I from diverse metazoan invertebrates. Molecular marine biology and biotechnology, 3, 294-299.

Galan, M., Pons, J.-B., Tournayre, O., Pierre, É., Leuchtmann, M., Pontier, D. \& Charbonnel, N. (2018). Metabarcoding for the parallel identification of several hundred predators and their prey: Application to bat species diet analysis. Molecular ecology resources, 14, 1-16.

Geller, J., Meyer, C., Parker, M. \& Hawk, H. (2013). Redesign of PCR primers for mitochondrial cytochrome coxidase subunit I for marine invertebrates and application in all-taxa biotic surveys. Molecular ecology resources, 13, 851-861.

Gibson, J.F., Shokralla, S., Curry, C., Baird, D.J., Monk, W.A., King, I. \& Hajibabaei, M. (2015). Large-Scale Biomonitoring of Remote and Threatened Ecosystems via High-Throughput Sequencing (D. Fontaneto, Ed.). PloS one, 10, e0138432-15.

Gibson, J., Shokralla, S., Porter, T.M., King, I., van Konynenburg, S., Janzen, D.H., Hallwachs, W. \& Hajibabaei, M. (2014). Simultaneous assessment of the macrobiome and microbiome in a bulk sample of tropical arthropods through DNA metasystematics. Proceedings of the National Academy of Sciences, 111, 8007-8012.

Hajibabaei, M., Janzen, D.H., Burns, J.M., Hallwachs, W. \& Hebert, P.D.N. (2006). DNA barcodes distinguish species of tropical Lepidoptera. Proceedings of the National Academy of Sciences of the United States of America, 103, 968-971.

Hajibabaei, M., Porter, T.M., Wright, M. \& Rudar, J. (2019a). COI metabarcoding primer choice affects richness and recovery of indicator taxa in freshwater systems. bioRxiv, 11, 2639-30.

Hajibabaei, M., Porter, T., Robinson, C. \& BioRxiv, D.B. (2019b). Watered-down biodiversity? A comparison of metabarcoding results from DNA extracted from matched water and bulk tissue biomonitoring samples. biorxiv.org

Hajibabaei, M., Shokralla, S., Zhou, X., Singer, G. \& Baird, D.J. (2011). Environmental Barcoding: A Next-Generation Sequencing Approach for Biomonitoring Applications Using River Benthos. PloS one, 6, 1-7.

Hajibabaei, M., Spall, J.L., Shokralla, S. \& van Konynenburg, S. (2012). Assessing biodiversity of a freshwater benthic macroinvertebrate community through non-destructive environmental barcoding of DNA from preservative ethanol. BMC ecology, 12, 1-1.

Hebert, P.D.N., Cywinska, A., Ball, S.L. \& deWaard, J.R. (2003). Biological identifications through DNA barcodes. Proceedings of the Royal Society B: Biological Sciences, 270, 313321. 
665

Hebert, P.D.N., Penton, E.H., Burns, J.M., Janzen, D.H. \& Hallwachs, W. (2004). Ten species in one: DNA barcoding reveals cryptic species in the neotropical skipper butterfly Astraptes fulgerator. Proceedings of the National Academy of Sciences of the United States of America, 101, 14812-14817.

Hernández-Triana, L.M., Prosser, S.W., Rodríguez-Perez, M.A., Chaverri, L.G., Hebert, P.D.N. \& Ryan Gregory, T. (2014). Recovery of DNA barcodes from blackfly museum specimens (Diptera: Simuliidae) using primer sets that target a variety of sequence lengths. Molecular ecology resources, 14, 508-518.

Hurlbert, S.H. (1984). Pseudoreplication and the Design of Ecological Field Experiments. Ecological Monographs, 54, 187-211.

Jusino, M.A., Banik, M.T., Palmer, J.M., Wray, A.K., Xiao, L., Pelton, E., Barber, J.R., Kawahara, A.Y., Gratton, C., Zachariah Peery, M. \& Lindner, D.L. (2018). An improved method for utilizing high-throughput amplicon sequencing to determine the diets of insectivorous animals. Molecular ecology resources.

Krehenwinkel, H., Fong, M., Kennedy, S., Huang, E.G., Noriyuki, S., Cayetano, L. \& Gillespie, R. (2018). The effect of DNA degradation bias in passive sampling devices on metabarcoding studies of arthropod communities and their associated microbiota (M. Kuntner, Ed.). PloS one, 13, e0189188-14.

Krehenwinkel, H., Wolf, M., Lim, J.Y., Rominger, A.J., Simison, W.B. \& Gillespie, R.G. (2017). Estimating and mitigating amplification bias in qualitative and quantitative arthropod metabarcoding. Scientific Reports, 1-12.

Leray, M. \& Knowlton, N. (2015). DNA barcoding and metabarcoding of standardized samples reveal patterns of marine benthic diversity. Proceedings of the National Academy of Sciences, 112, 2076-2081.

Leray, M. \& Knowlton, N. (2017). Random sampling causes the low reproducibility of rare eukaryotic OTUs in Illumina COI metabarcoding. PeerJ, 5, e3006-27.

Leray, M., Yang, J.Y., Meyer, C.P., Mills, S.C., Agudelo, N., Ranwez, V., Boehm, J.T. \& Machida, R.J. (2013). A new versatile primer set targeting a short fragment of the mitochondrial COI region for metabarcoding metazoan diversity: application for characterizing coral reef fish gut contents. Frontiers in zoology, 10, 1-14.

Macher, J.N. \& Weigand, A.M. (2018). A DNA metabarcoding protocol for hyporheic freshwater meiofauna: Evaluating highly degenerate COI primers and replication strategy. Metabarcoding and Metagenomics, 2, 63-15.

Macher, J.N., Vivancos, A., Piggott, J.J., Centeno, F.C., Matthaei, C.D. \& Leese, F. (2018). Comparison of environmental DNA and bulk-sample metabarcoding using highly degenerate cytochrome coxidase I primers. Molecular ecology resources, 17, 1231-43.

Marquina, D., Andersson, A.F. \& Ronquist, F. (2018). New mitochondrial primers for 
metabarcoding of insects, designed and evaluated using in silicomethods. Molecular ecology resources, 1-39.

Martin, M. (2011). Cutadapt removes adapter sequences from high-throughput sequencing reads. EMBnet journal, 17, 10-12.

Meusnier, I., Singer, G.A., Landry, J.-F., Hickey, D.A., Hebert, P.D. \& Hajibabaei, M. (2008). A universal DNA mini-barcode for biodiversity analysis. BMC genomics, 9, 214.

Meyer, C.P. (2003). Molecular systematics of cowries (Gastropoda: Cypraeidae) and diversification patterns in the tropics. Biological Journal of the Linnean Society, 79, 401-459.

Nichols, R.V., Vollmers, C., Newsom, L.A., Wang, Y., Heintzman, P.D., Leighton, M., Green, R.E. \& Shapiro, B. (2018). Minimizing polymerase biases in metabarcoding. Molecular ecology resources, 12, R18-13.

Park, D.-S., Foottit, R., Maw, E. \& Hebert, P.D.N. (2011). Barcoding Bugs: DNA-Based Identification of the True Bugs (Insecta: Hemiptera: Heteroptera) (M.T.P. Gilbert, Ed.). PloS one, 6, e18749-9.

Piñol, J., Mir, G., Gomez-Polo, P. \& Agustí, N. (2014). Universal and blocking primer mismatches limit the use of high-throughput DNA sequencing for the quantitative metabarcoding of arthropods. Molecular ecology resources, 15, 1-12.

Piñol, J., Senar, M.A. \& Symondson, W.O.C. (2018). The choice of universal primers and the characteristics of the species mixture determine when DNA metabarcoding can be quantitative. Molecular Ecology, 6, 1809-13.

Porter, T.M. \& Hajibabaei, M. (2018a). Automated high throughput animal CO1 metabarcode classification. Scientific Reports, 1-10.

Porter, T.M. \& Hajibabaei, M. (2018b). Over 2.5 million COI sequences in GenBank and growing (W. Arthofer, Ed.). PloS one, 13, e0200177-16.

Prosser, S.W.J., deWaard, J.R., Miller, S.E. \& Hebert, P.D.N. (2015). DNA barcodes from century-old type specimens using next-generation sequencing. Molecular ecology resources, 16, 487-497.

Ratnasingham, S. \& Hebert, P. (2007). BOLD: The Barcode of Life Data System (http://www. barcodinglife. org). Molecular Ecology Notes, 7, 355-364.

Ratnasingham, S. \& Hebert, P.D.N. (2013). A DNA-Based Registry for All Animal Species: The Barcode Index Number (BIN) System (D. Fontaneto, Ed.). PloS one, 8, e66213-16.

Reuter, J.A., Spacek, D.V. \& Snyder, M.P. (2015). High-Throughput Sequencing Technologies. Molecular Cell, 58, 586-597.

Sellers, G.S., Di Muri, C., Gómez, A. \& Hänfling, B. (2018). Mu-DNA: a modular universal DNA 
746

747

748

extraction method adaptable for a wide range of sample types. Metabarcoding and Metagenomics, 2, e24556-11.

Shokralla, S., Porter, T.M., Gibson, J.F., Dobosz, R., Janzen, D.H., Hallwachs, W., Golding, G.B. \& Hajibabaei, M. (2015). Massively parallel multiplex DNA sequencing for specimen identification using an Illumina MiSeq platform. Scientific Reports, 5, 9687.

Singer, G. A. C., Fahner, N. A., Barnes, J. G., McCarthy, A., \& Hajibabaei, M. (2019). Comprehensive biodiversity analysis via ultra-deep patterned flow cell technology: a case study of eDNA metabarcoding seawater. Scientific Reports, 9(1), 5991.

Smith, D.P. \& Peay, K.G. (2014). Sequence Depth, Not PCR Replication, Improves Ecological Inference from Next Generation DNA Sequencing (C.A. Kellogg, Ed.). PloS one, 9, e90234.

Steffen, W., Richardson, K., Rockström, J., Cornell, S.E., Fetzer, I., Bennett, E.M., Biggs, R., Carpenter, S.R., de Vries, W., de Wit, C.A., Folke, C., Gerten, D., Heinke, J., Mace, G.M., Persson, L.M., Ramanathan, V., Reyers, B. \& Sörlin, S. (2015). Planetary boundaries: guiding human development on a changing planet. Science (New York, NY), 347, 1259855.

Vamos, E.E., Elbrecht, V. \& Leese, F. (2017). Short COI markers for freshwater macroinvertebrate metabarcoding. Metabarcoding and Metagenomics, 1-20.

Vierna, J., Doña, J., Vizcaíno, A., Serrano, D. \& Jovani, R. (2017). PCR cycles above routine numbers do not compromise high-throughput DNA barcoding results. (F. Chain, Ed.). Genome, 60, 868-873.

Vörösmarty, C.J., McIntyre, P.B., Gessner, M.O., Dudgeon, D., Prusevich, A., Green, P., Glidden, S., Bunn, S.E., Sullivan, C.A., Liermann, C.R. \& Davies, P.M. (2010). Global threats to human water security and river biodiversity. Nature, 467, 555-561.

Wangensteen, O.S., Palacín, C., Guardiola, M. \& Turon, X. (2018). DNA metabarcoding of littoral hard-bottom communities: high diversity and database gaps revealed by two molecular markers. PeerJ, 6, e4705-30.

Yu, D.W., Ji, Y., Emerson, B.C., Wang, X., Ye, C., Yang, C. \& Ding, Z. (2012). Biodiversity soup: metabarcoding of arthropods for rapid biodiversity assessment and biomonitoring. Methods in Ecology and Evolution, 3, 613-623.

Zeale, M.R.K., Butlin, R.K., Barker, G.L.A., Lees, D.C. \& Jones, G. (2011). Taxon-specific PCR for DNA barcoding arthropod prey in bat faeces. Molecular ecology resources, 11, 236-244.

Zepeda-Mendoza, M.L., Bohmann, K., Baez, A.C. \& Gilbert, M.T.P. (2016). DAMe: a toolkit for the initial processing of datasets with PCR replicates of double-tagged amplicons for DNA metabarcoding analyses. BMC Research Notes, 1-13.

Zhang, G.K., Chain, F.J.J., Abbott, C.L. \& Cristescu, M.E. (2018). Metabarcoding using multiplexed markers increases species detection in complex zooplankton communities. Evolutionary Applications, 215, 403-40. 


\section{Figure 1}

Overview of the experimental design.

The performance of 36 primer pairs was tested via gradient PCRs with a mock community of insects (A). The 21 pairs that showed best amplification results (B) were selected for further DNA metabarcoding runs utilizing both the mock community and a Malaise trap sample (C). Based on the metabarcoding results, four primer sets showing the good performance were selected for a third test that examined the effects of varying annealing temperatures on taxon recovery and non-specific amplification (D). Based on all results, the optimal primer sets were designated $(E)$. 


\section{Experimental outline}

- 36 Candidate primer sets (Fig. 2, primer sequences Tab. S2)

- Two insect bulk samples for testing:

374 taxa mock insect community

One complete malaise trap,

(from Braukmann et al. 2019) 1 week July, Ontario, Canada

\section{Primer shortlist}

- Gradient PCR, annealing $46-64.5^{\circ} \mathrm{C}$ (Fig. S7 \& S8)

- Discarding primer sets with poor amplification or no plateau in PCR gradient

\section{Metabarcoding $\left(46^{\circ} \mathrm{C}\right.$ annealing)}

- Fusion primer design for 21 primer sets

- Two step PCR at $46^{\circ}$ annealing temperature

- Two Illumina MiSeq sequencing runs (v3 300 bp Paired End):

Run I:"mock community" Run II: "malaise trap sample"

- Objective: Assess and compare performance of different primer sets (Fig. $3 \& 4$ )

\section{Mock sample gradient metabarcoding}

- Fusion primer design for 4 representative primer sets

- Two step PCR at different annealing temperatures:

$40.0,41.6,43.7,46.0,48.5,50.8,53.0,54.7$ and $56.0^{\circ} \mathrm{C}$

- MiSeq run III: "gradient"

- Objective: Optimise annealing temperature for the best performing primer sets (Fig. 5)

\section{Primer recommendations}




\section{Figure 2}

Target and amplicon length for the 36 primer sets evaluated via gradient PCR.

The 21 primer sets selected for sequencing are highlighted in yellow while an ID for each pair is shown on the left. Primer references: (Folmer et al. 1994; Meyer 2003; Hebert et al. 2004; Hajibabaei et al. 2006; Meusnier et al. 2008; Hajibabaei et al. 2011; Zeale et al. 2011; Park et al. 2011; Yu et al. 2012; Hajibabaei et al. 2012; Leray et al. 2013; Geller et al. 2013; Gibson et al. 2014; Hernández-Triana et al. 2014; Shokralla et al. 2015; Brandon-Mong et al. 2015; Gibson et al. 2015; Prosser et al. 2015; Elbrecht \& Leese 2017; Vamos et al. 2017; Wangensteen et al. 2018; Galan et al. 2018) . 


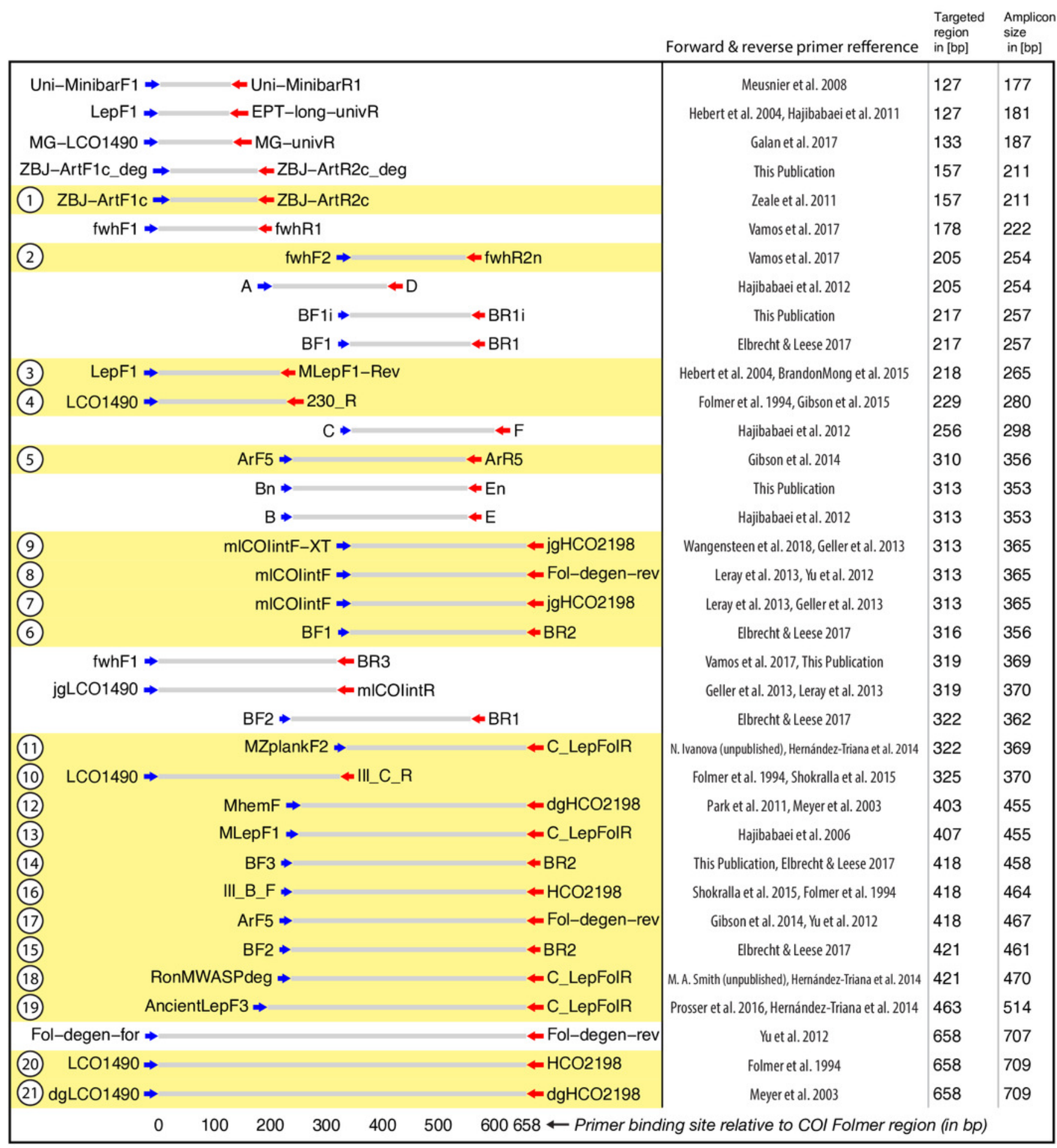




\section{Figure 3}

Proportion of sequences discarded or mapped to reference sequences in the mock community.

A: Bar plots show the relative proportion of reads that were discarded or mapped. Numbers in bars indicate the proportion of reads that matched one of the 374 species in the mock community. The number for each primer pair on the x-axis corresponds with that in Figure 2.

B: Proportion of sequences discarded by max expected errors = 1 filtering using Usearch, plotted against the length of the target region (in bp). Red line indicates linear regression. 

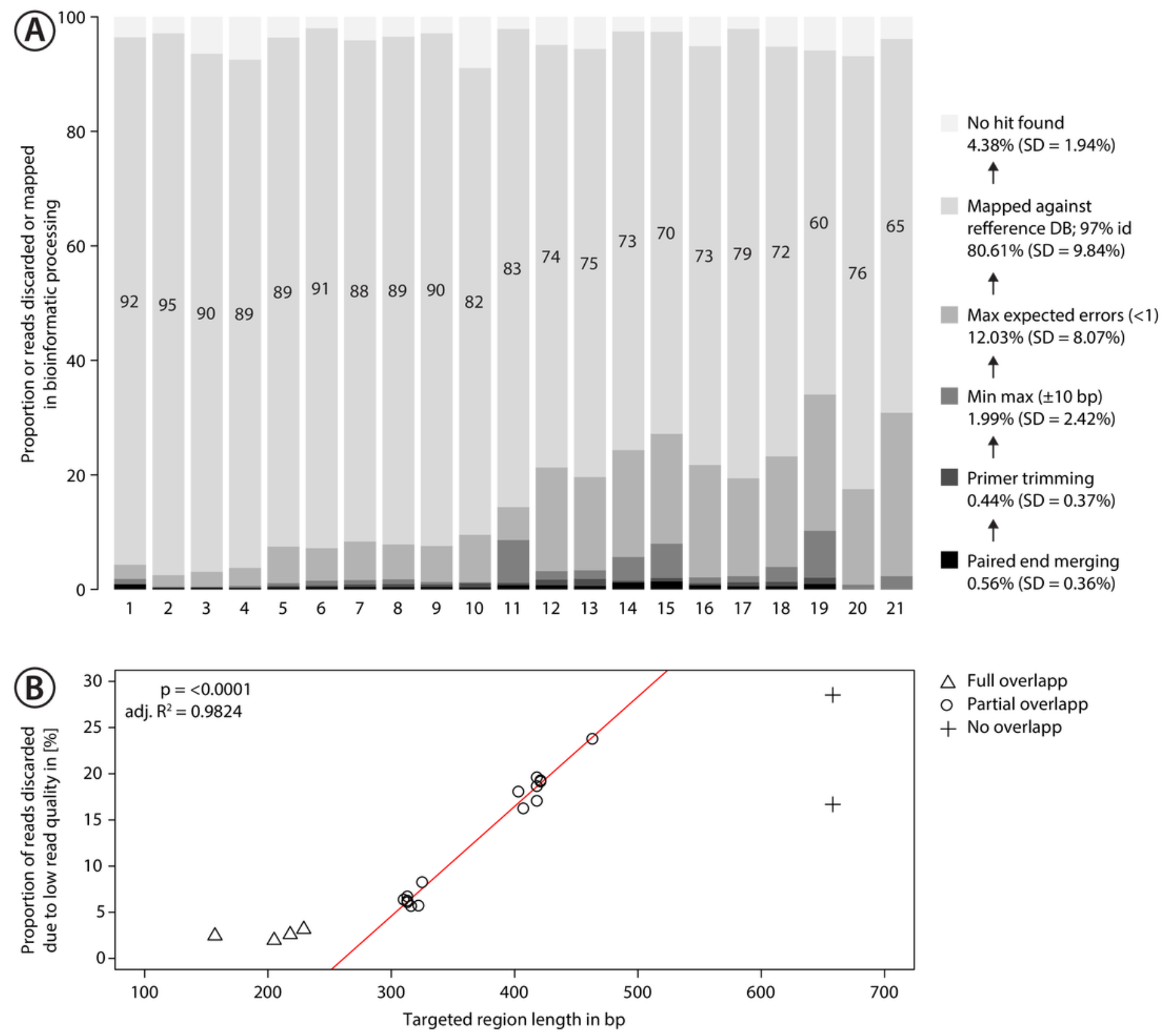

$\triangle$ Full overlapp

○ Partial overlapp

+ No overlapp 


\section{Figure 4}

Bar plot showing the number of BINs recovered using metabarcoding with 21 primer pairs.

The dark grey bar indicates subsampling at 10,000 reads while the light grey bar indicates subsampling at 100,000 reads per sample, each run with 1,000 replicates. Error bars show the standard deviation. Primer combinations affected by primer slippage (Elbrecht \& Steinke 2018) are marked with a red X. A: Mock sample data, with primer combinations highlighted in green that detected more than 350 of the 374 BINs, while those that recovered fewer than 310 BINS are highlighted in red. B: Malaise trap data - primer combinations highlighted in green detected more than 750 BINs while those highlighted in red detected less than 600 BINs. 

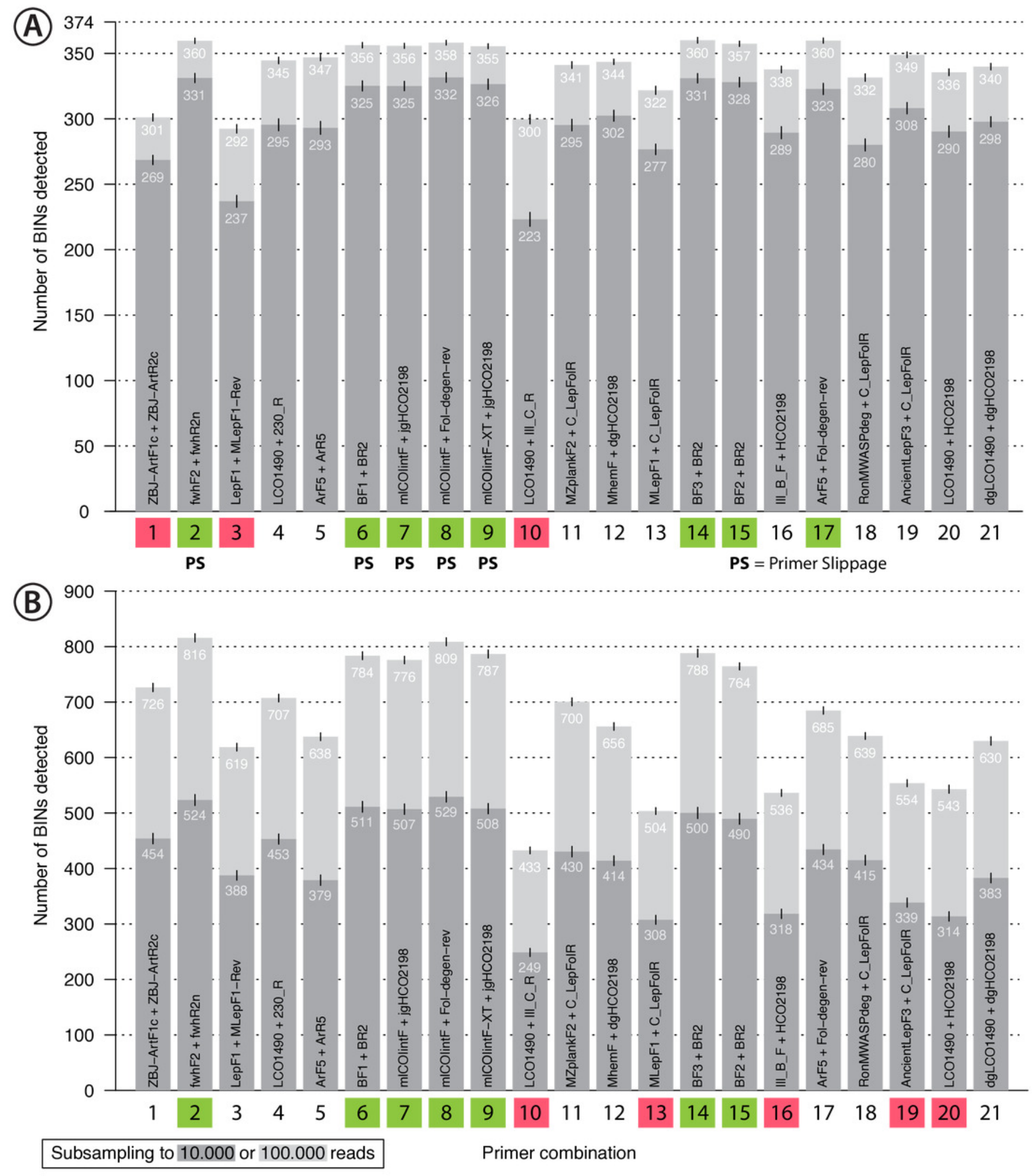


\section{Figure 5}

Bar plot showing the number of BINs recovered from the mock community at different annealing temperatures.

The dark grey bar indicates subsampling at 10,000 reads, while the light grey bar depicts subsampling at 100,000 reads per samples; both were run with 1,000 replicates. Error bars show the standard deviation.

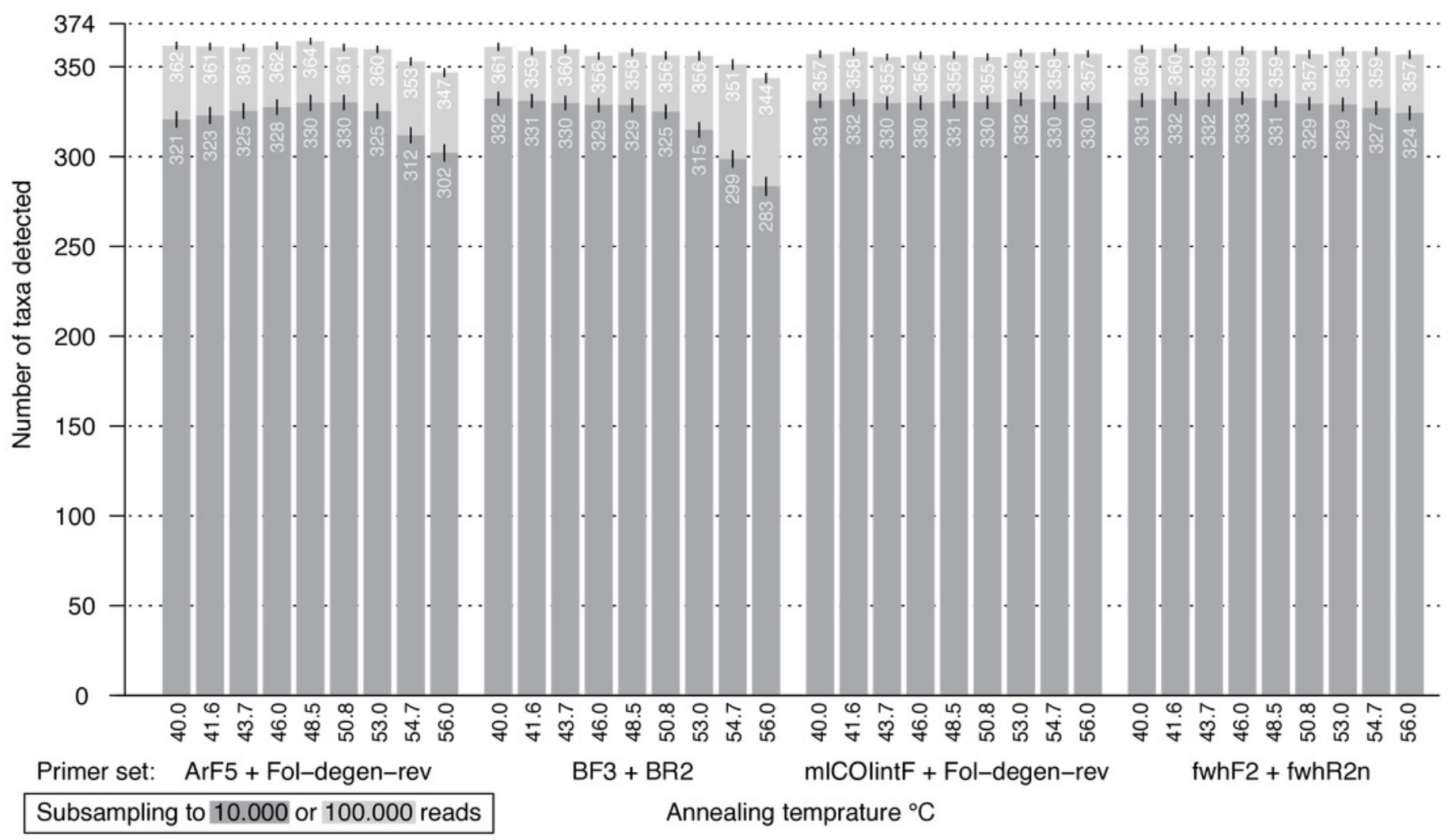

